\title{
In situ diffraction studies of the initial growth processes of textured icosahedral quasicrystalline thin films
}

\author{
P. R. Willmott, ${ }^{1, *}$ C. M. Schlepütz, ${ }^{1}$ R. Herger, ${ }^{1}$ B. D. Patterson,${ }^{1}$ K. Hassdenteufel, ${ }^{2}$ and W. Steurer ${ }^{2}$ \\ ${ }^{1}$ Swiss Light Source, Paul Scherrer Institute, CH-5232 Villigen, Switzerland \\ ${ }^{2}$ Laboratory of Crystallography, Department of Materials, ETH-Hönggerberg, CH-8093 Zürich, Switzerland
}

(Received 19 October 2004; published 14 March 2005)

\begin{abstract}
The seeding and morphology of quasicrystalline $\mathrm{Ti}_{x} \mathrm{Ni}_{y} \mathrm{Zr}_{1-x-y}$ thin films grown by pulsed-laser deposition on $\mathrm{Al}_{2} \mathrm{O}_{3}(0001)$ have been investigated in situ, using in-plane $\mathrm{x}$-ray diffraction and reflectivity with synchrotron radiation. The crystallinity of the final films was further studied ex situ using a laboratory x-ray source. Local icosahedral order becomes established after a film thickness of $1.5 \mathrm{~nm}$, which is followed by an abrupt change in the growth rate after a film thickness of approximately $5 \mathrm{~nm}$, as long-range quasicrystalline order begins to become energetically favorable. The films grow two-dimensionally even up to thicknesses of over $100 \mathrm{~nm}$ and are highly textured, consisting of columnar grains with one of their fivefold symmetry axes perpendicular to the substrate surface.
\end{abstract}

DOI: $10.1103 /$ PhysRevB.71.094203

PACS number(s): 61.44.Br, 61.10.Nz, 68.55.Ac, 81.15.Fg

\section{INTRODUCTION}

Quasicrystals (QCs) have interesting physical and chemical properties: many have conductivities typical of semiconductors, are very resistant to corrosion, have high hardnesses, and low coefficients of friction. These last two properties make QCs especially interesting as protective and tribological coatings, particularly because bulk QCs tend to be highly brittle. ${ }^{1}$ In addition, Ti-based QCs are able to reversibly store large quantities of hydrogen, making them potential candidates for solid-state or microintegrated batteries. ${ }^{2}$

Nucleation phenomena in icosahedral QC thin films were studied $^{3-5}$ as early as 1987. Reactive diffusion, responsible for the development of the icosahedral structure, was analyzed using electron microscopy in the specific case of $\mathrm{Al}$ - Mn QC thin films prepared by sequential deposition of $\mathrm{Al}$ and $\mathrm{Mn}$. There have also been studies of the nucleation of the QC phase by interdiffusion of annealed multilayers, ${ }^{3}$ in which both the icosahedral and decagonal phases were identified, ${ }^{6}$ their relative contribution depending on the initial multilayer structure and heat treatment.

Nonetheless, there have been essentially no in situ structural investigations during growth of the initiation of quasicrystalline order in thin films on crystalline substrates, which may proceed quite differently from bulk material, due to the confined extent in the growth direction and the potentially different kinetics of the chosen deposition technique (see below). We have undertaken such studies by exploiting, on the one hand, the high brightness of synchrotron radiation, which allows one to probe only the upper few nanometers using grazing-incidence diffraction techniques. Secondly, we have used pulsed-laser deposition (PLD) to grow icosahedral $\mathrm{Ti}_{x} \mathrm{Ni}_{y} \mathrm{Zr}_{1-x-y}(i-\mathrm{TNZ})$ thin films in situ. ${ }^{7}$ Importantly, PLD's high instantaneous flux and consequent high degree of supersaturation should promote the QC phase over related crystalline phases, while the energy regime of the incident particles enhances surface diffusion. ${ }^{8-12}$ In addition, PLD is eminently suited to experiments in which film growth is repeatedly interrupted in order to analyze the deposit, because of the ease of supplying arbitrarily small doses of deposition flux with essentially instantaneous rising and falling flanks. $i$-TNZ was chosen, as it does not contain $\mathrm{Al}$, the most common element found in QCs, which produces large amounts of unwanted macroscopic particulates under nanosecond laser radiation. ${ }^{13}$

\section{EXPERIMENT}

The experimental setup has been detailed elsewhere. ${ }^{7,14}$ Briefly, a UHV PLD chamber has been designed to fit on a large $(2+3)$ circle diffractometer ${ }^{15}$ at the Materials Science beamline of the Swiss Light Source synchrotron radiation facility. ${ }^{16}$ The chamber walls include a large beryllium section, which is transparent to the $1 \AA \mathrm{x}$ radiation used for the in situ experiments. Material is ablated using a frequencyquadrupled $\mathrm{Nd}$ :YAG laser $\left(5 \mathrm{~ns}, 2.5 \mathrm{~J} \mathrm{~cm}^{-2}, 10 \mathrm{~Hz}\right.$ ). The $\mathrm{Al}_{2} \mathrm{O}_{3}(0001)$ substrate is placed $60 \mathrm{~mm}$ from the ablation target and heated by pressing it to an ohmically heated $\mathrm{Si}$ wafer. The chosen deposition temperature was $435 \pm 5^{\circ} \mathrm{C}$, which, in a set of ex situ preliminary experiments, was found to be the highest temperature for which the films contained no or insignificant evidence of a crystalline phase (e.g., the Laves phase), and which produced the sharpest QC-phase diffraction peaks. No postannealing of the films was necessary.

By using a segmented-rod ablation target, ${ }^{7}$ the composition of the film $(x$ and $y$ ) can be varied at will. This also allows the use of inexpensive, high-purity elemental target material with low intrinsic oxygen content, which is especially important for the synthesis of Ti-based QC alloys, as even oxygen partial pressures as low as a few hundred parts per million can stabilize the $\mathrm{Ti}_{2} \mathrm{Ni}$ phase and eliminate both the QC and Laves phases. ${ }^{17}$ Large bulk QC alloy targets are difficult to produce, ${ }^{18}$ and noncongruent ablation often leads to initial enrichment of one component on the target surface, requiring a long pre-ablation period before steady state is achieved. ${ }^{11}$ This problem is circumvented if each elemental component is ablated sequentially. The reproducibility of the final film composition between experiments was limited to 

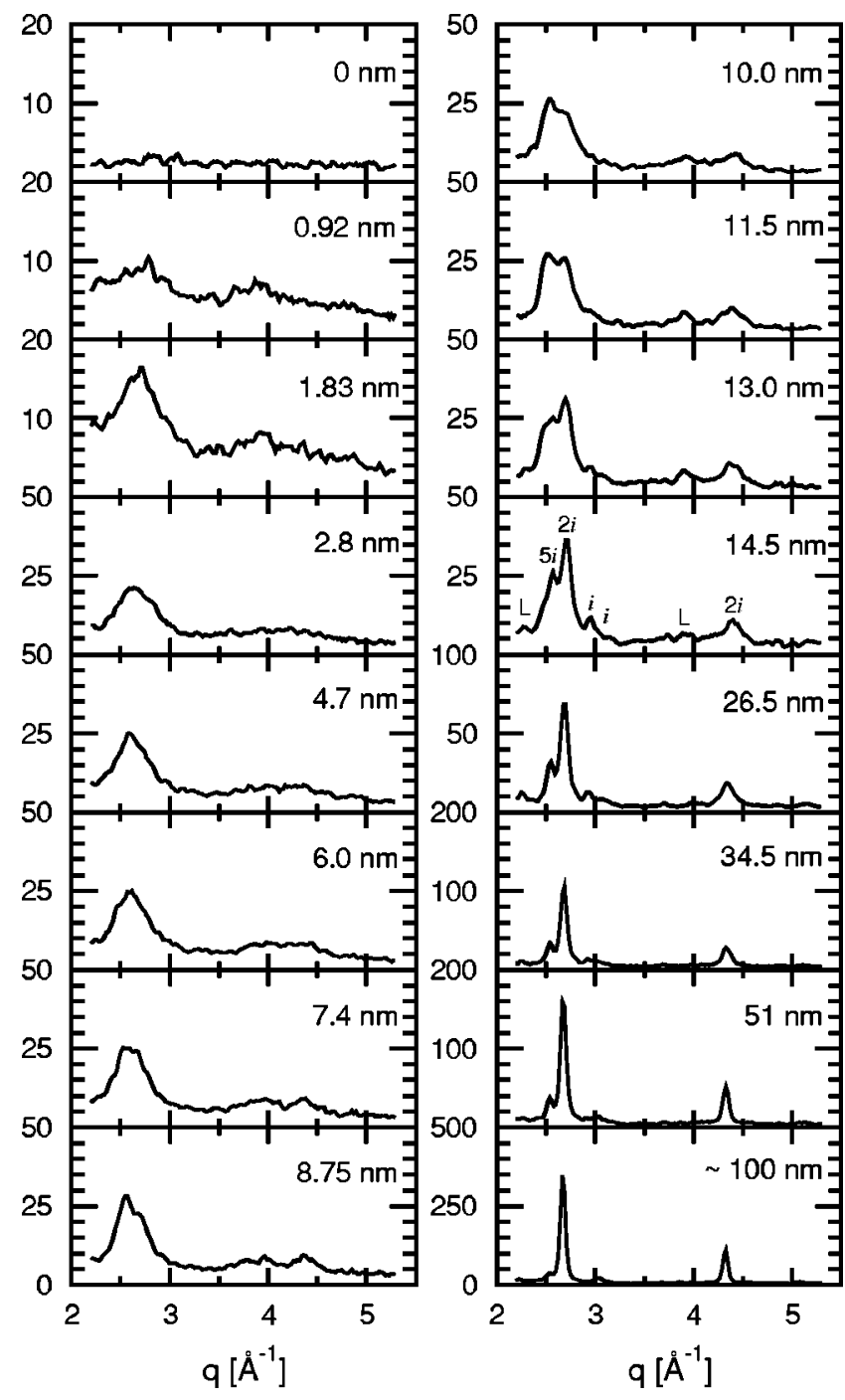

FIG. 1. $2 \theta$ in-plane $x$-ray diffraction patterns of $\mathrm{Ti}_{x} \mathrm{Ni}_{y} \mathrm{Zr}_{1-x-y}$ thin films as a function of the momentum transfer $q=4 \pi \sin \theta / \lambda$ and film thickness. Spectral intensities between different film thicknesses can be directly compared. The spectrum for the $14.5 \mathrm{~nm}$ film is labeled, as it contains important features, including the in-plane equivalent (100000) fivefold axis reflection (5i), the $(01 \overline{1000)}$ and $(0100 \overline{1} 0)$ twofold reflections $(2 i)$, two further weak reflections $(i)$ associated with the icosahedral phase, and the two Laves phases (L).

approximately \pm 1 percentile, as determined by Rutherford backscattering (RBS). All films had a small fraction of a percent of $\mathrm{Hf}$, as this represents the major impurity in the $\mathrm{Zr}$ target section. ${ }^{7}$

\section{RESULTS AND DISCUSSION}

The evolution of the in-plane crystallinity of a film with the composition $\mathrm{Ti}_{0.385} \mathrm{Ni}_{0.186} \mathrm{Zr}_{0.427} \mathrm{Hf}_{0.002}$, close to the ideal QC composition, ${ }^{18}$ as a function of $t$, the film thickness (see below), is shown in the diffractograms in Fig. 1. The peaks were identified using the QUAREF program ${ }^{19}$ using the quasicrystalline lattice constant $a=2.4456 \AA$, and were in agree- ment with literature values ${ }^{20}$ to within $\pm 0.01 \AA^{-1}$. The angle of incidence of the X-ray beam was $0.15 \pm 0.02^{\circ}$, below the critical angles for total reflection of sapphire and $i$-TNZ of $0.185^{\circ}$ and $0.22^{\circ}$, respectively; hence, only the topmost $3.2 \pm 0.05 \mathrm{~nm}$ layer of the film is probed, corresponding to the penetration depth of the evanescent wave.

After the growth of $\approx 1.5 \mathrm{~nm}$, a broad diffraction feature becomes clearly visible, centered at $q=2.65 \AA^{-1}$, with a full width at half-maximum (FWHM) of $0.4 \AA^{-1}$. Ignoring strain effects, this corresponds to an average characteristic size of only $1.5 \mathrm{~nm}$. A still broader but weaker feature centered at $\approx 4.2 \AA^{-1}$ can also be seen. This diffraction pattern is essentially identical to that demonstrating local icosahedral order (LIO) in the undercooled liquid phase of $\mathrm{Ti}_{x} \mathrm{Ni}_{y} \mathrm{Zr}_{1-x-y}$ presented by Kelton et al. ${ }^{20}$ in their study validating the hypothesis of supercooling in metals proposed by Frank. ${ }^{21} i$-TNZ is a Bergman-type QC, and the diameter of the smallest Bergman cluster (containing 45 atoms) is calculated ${ }^{22}$ to be approximately $d_{0}=1.4 \mathrm{~nm}$, in good agreement with the width of the strongest feature.

From here on, until a thickness of $t \approx 6 \mathrm{~nm}$, there is little change in the intensities or shapes of these two features, indicative of continued growth of an amorphous layer containing LIO. Similar initial layers have also been identified using electron microscopy by Widjaja and Marks in sputter deposition of decagonal $\mathrm{Al}-\mathrm{Cu}-\mathrm{Fe}-\mathrm{Cr}$ grown on sapphire ${ }^{23}$ and by Brien et al. ${ }^{12}$ in PLD of $i$-TNZ.

From $t \approx 6 \mathrm{~nm}$ to $14 \mathrm{~nm}$, long-range icosahedral order becomes evident as the feature at $2.65 \AA^{-1}$ resolves into the in-plane equivalent of the fivefold (100000) $i$-phase reflection $^{24}$ at $2.57 \AA^{-1}$ and the twofold (01-1000) $i$-phase reflection at $2.70 \AA^{-1}$, while the broader feature centered at $4.1 \AA^{-1}$ sharpens into the second twofold $(0100 \overline{10}) i$-phase reflection at $4.34 \AA^{-1}$. This transformation begins at a film thickness consistent with the size of the next hierarchical inflation $^{25}$ of the Bergman cluster of about $d_{1}=\tau^{3} d_{0} \approx 6 \mathrm{~nm}$, whereby $\tau$ is the golden ratio $(1+\sqrt{5}) / 2$. Seeding of the quasicrystalline phase was also tentatively identified at around this thickness by Brien et al. ${ }^{12}$ from electron microscopy images. By $t=14.5 \mathrm{~nm}$, the peak widths indicate an average in-plane QC size of a little over $5 \mathrm{~nm}$. Additional peaks at 2.28 and $3.8 \AA^{-1}$ can be attributed to the C14 Laves phase, although these essentially disappear again by $t=35 \mathrm{~nm}$.

$\mathrm{Up}$ to $t=14.5 \mathrm{~nm}$, the QC reflections have structure factors similar to those found in powder spectra. From here on, however, only the two reflections associated with the inequivalent twofold axes increase in intensity in the in-plane spectra, as this orientation grows to the exclusion of crystallites of other orientations, until at $100 \mathrm{~nm}$, they comprise over $98 \%$ of the signal. The progression of the integrated intensities of $5 i$ and the $2 i$ reflections that emerge from the initial strong feature, together with their linewidths, is summarized in Fig. 2.

The in-plane twofold axes are perpendicular to a fivefold axis in the growth direction, and indeed these reflections completely dominate ex situ out-of-plane $\theta / 2 \theta$ spectra of the final films (see Fig. 3). The FWHMs correspond to grain sizes in-plane of $\approx 10 \mathrm{~nm}$ and out-of-plane of $\approx 60 \mathrm{~nm}$.

The in-plane $2 \theta$ spectra do not depend on the rotation angle of the film about its surface normal (i.e., $\omega$ ), indicating 


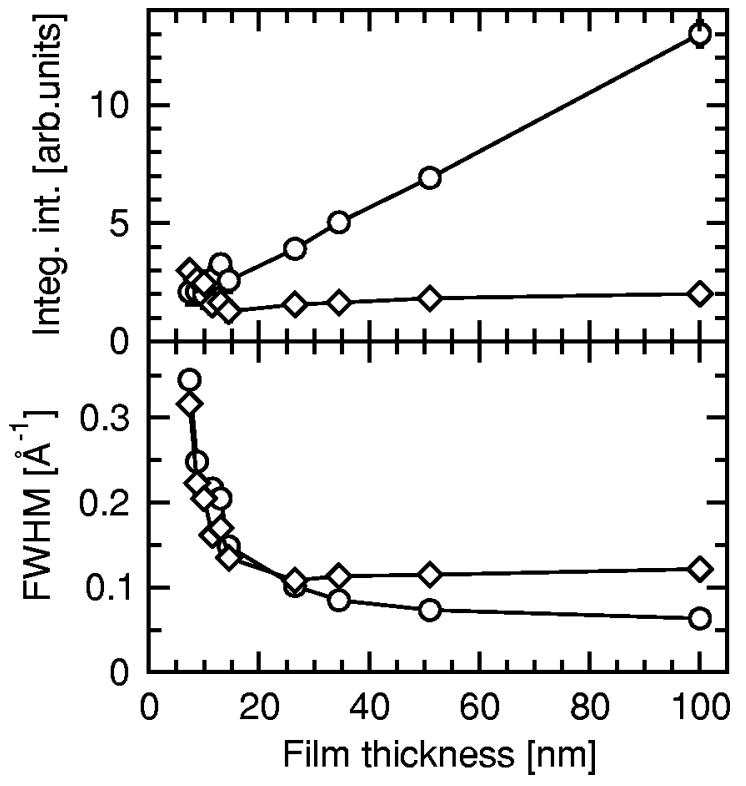

FIG. 2. Upper panel: the integrated intensities of the $5 i$ and $2 i$ in-plane reflections emerging from the initial broad feature at $2.65 \AA^{-1}$ as a function of the film thickness. Lower panel: The FWHMs $=\sigma \sqrt{8 \ln 2}$ of the fitted Gaussians used to determine the data in the upper panel, whereby $I=I_{0} \exp \left[-\left(q-q_{0}\right)^{2} / 2 \sigma^{2}\right]$; $\diamond$, fivefold; $\bigcirc$, twofold.

that the films consist of QC grains with the fivefold axis pointing in the growth direction, but with random orientation of the twofold axes in-plane. This was confirmed by recording pole figures for the fivefold and twofold reflections, which show rings at the expected angles (inset, Fig. 3). This is in contrast to the results of Brien et al., ${ }^{11}$ for which the fivefold axis is tilted to the sapphire substrate surface by an angle of $6.4^{\circ}$, which they tentatively attribute to the non-

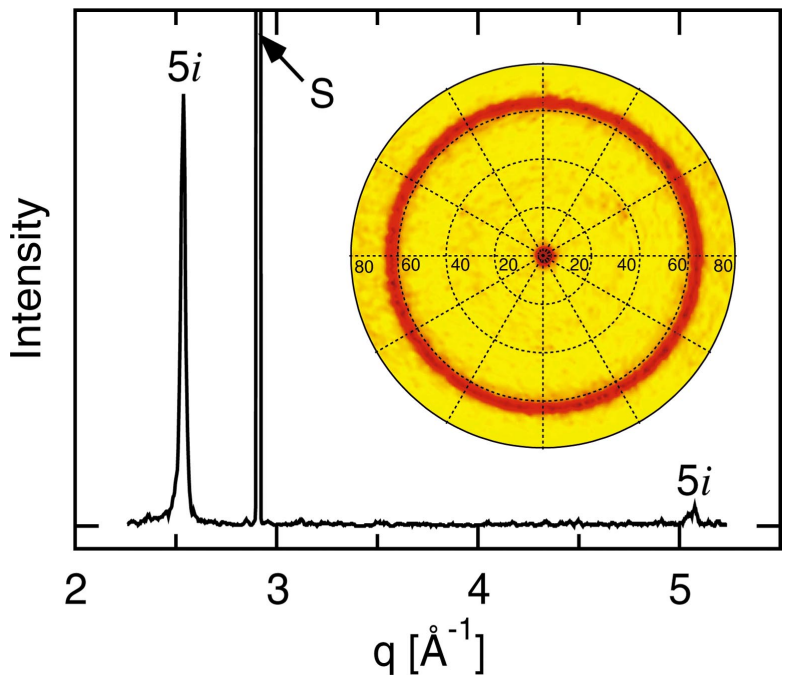

FIG. 3. (Color) Out-of plane diffraction pattern of the thickest $(\sim 170 \mathrm{~nm}) i$-TNZ film, using $\mathrm{Cu} K_{\alpha}$ radiation plotted as a function of $q$. Other than the substrate peak (S), only the (100000) and (200000) fivefold reflections can be seen. Inset: pole figure of the (100000) fivefold reflection, showing the out-of-plane texture and a ring at the dodecahedral dihedral angle of $63.43^{\circ}$.

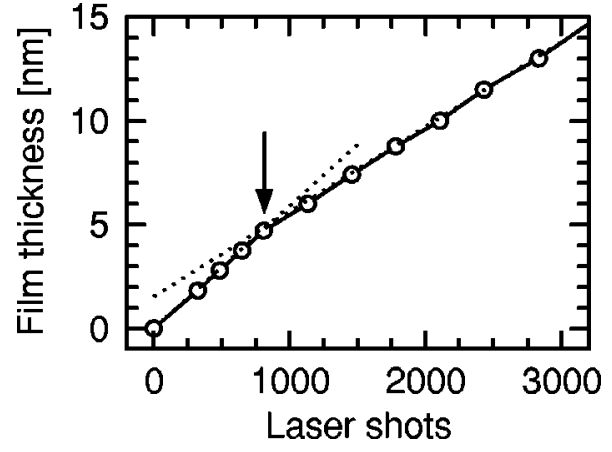

FIG. 4. The film thickness as determined by Kiessig fringes in the reflectivity curves as a function of the number of laser shots. The arrow indicates where the growth rate abruptly changes. The dotted lines are the best linear fits of the two gradients. The error in the thickness is around $\pm 0.2 \mathrm{~nm}$ up to thicknesses of approximately $4 \mathrm{~nm}$, and thereafter less than $\pm 0.1 \mathrm{~nm}$.

normal incident angle of the ablation plasma to the surface during growth. In our setup, the plasma also impinges on the substrate at an angle of approximately $10^{\circ}$, but we observe no similar inclination to the columnar texture.

A notable feature of the QC films is their flatness, manifested by clear Kiessig fringes in the reflectivity curves, ${ }^{7}$ even for thicknesses greater than $100 \mathrm{~nm}$, which exhibit typical roughnesses of the order of $1 \mathrm{~nm}$. This flatness has allowed us to accurately determine the film thickness in-situ during interrupted growth, as a function of laser shots (Fig. 4). The growth rate shows an abrupt reduction from $(5.8 \pm 0.1) \times 10^{-3} \mathrm{~nm}(\text { laser shot })^{-1} \quad$ to $\quad(4.1 \pm 0.05)$ $\times 10^{-3} \mathrm{~nm}$ (laser shot) ${ }^{-1}$ at approximately $5 \mathrm{~nm}$, close to the point where the $d_{1}$-Bergman cluster becomes viable (see above). This indicates that up to this thickness, the amorphous layer, although remaining atomically flat, has a more open structure and lower density $\left(\approx 4.2 \mathrm{~g} \mathrm{~cm}^{-3}\right.$ or $71 \%$ of the bulk density), perhaps with a high fraction of atomic voids, resulting in the higher growth rates. Recently, Kelton has presented extended x-ray absorption fine-structure spectra of different $\mathrm{Ti}-\mathrm{Ni}-\mathrm{Zr}$ alloys, ${ }^{26}$ in which the most amorphous structures (i.e., those with the least features in the extended fine structure) have nearest-neighbor separations some $10 \%$ larger than for the more (quasi)crystalline materials, in good agreement with our data. This change in density is confirmed in Fig. 5 by a steplike increase in the critical angle from $\alpha_{c} \approx 0.186^{\circ}$ for $t \leqslant 5 \mathrm{~nm}$ to $0.220^{\circ}$ for $t \geqslant 18 \mathrm{~nm}$, as the evanescent wave begins to probe only material with the density of bulk $i$-TNZ (approximately $5.95 \mathrm{~g} \mathrm{~cm}^{-3}$ ).

Growth of QCs by nucleation on a surface from a flux originating in the gas phase is different than, for example, Czochralski growth of bulk QCs from a melt. The high instantaneous deposition rates of PLD, of the order of 2000 (atomic layers) $\mathrm{s}^{-1}$, induce high nucleation rates. ${ }^{9}$ For growth temperatures well below the melt temperature $T_{m}$, the impinging particles will physisorb on the surface and transfer their kinetic energy (of the order of $25 \mathrm{eV}$ for PLD) to the local volume on the time scale of several picoseconds. Simulations indicate that this "thermal spike" results in local melting to a depth of a few atomic radii and also in enhanced surface diffusion over a few tens of picoseconds. ${ }^{27}$ This is 


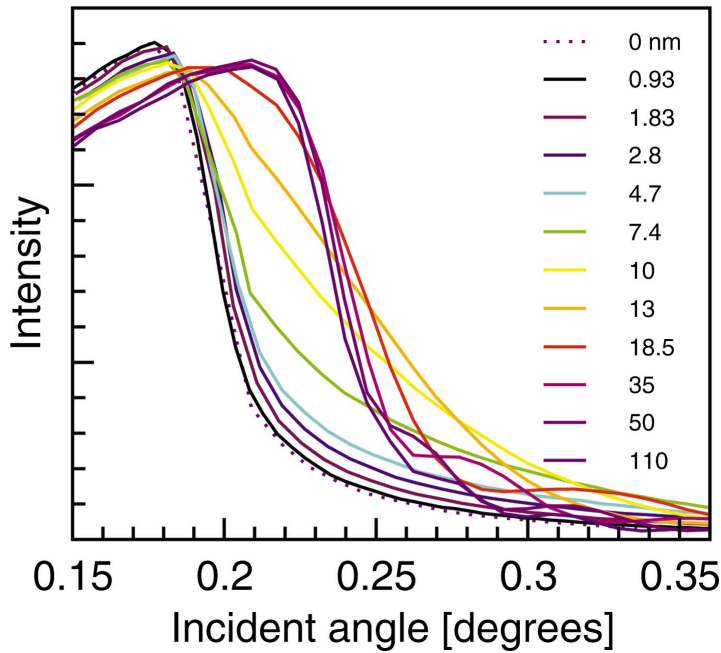

FIG. 5. (Color) The reflectivity around the critical angle as a function of film thickness.

one to two orders of magnitude greater than typical vibrational periods; hence, we can expect the adatom to hop a distance of $\sim \sqrt{100}=10$ atomic spacings before it is thermalized. More recent molecular dynamics calculations, in which local surface melting caused by an energetic collision cascade promotes diffusion over several picoseconds, yield similar values for the enhanced diffusion radius. ${ }^{28,29}$ This explains why postannealing of the films was unnecessary, in contrast to most other techniques applied to QC thin-film growth. ${ }^{9}$

Formation of the smallest Bergman clusters requires thermal motion over $1 \mathrm{~nm}$ or more, ${ }^{30}$ which is known to occur in the transient "melt" directly after impingement of hyperthermal species in PLD. Hence, the high nucleation rate and thermal spike associated with PLD should promote seeding of the icosahedral phase even at low substrate temperatures.

The flatness of our films is consistent with modern opinion on the development of surface morphologies of QCs. It is now thought that the role of energetically and mechanically stable clusters ${ }^{31,32}$ plays a secondary role to the energy minimization of the most closely packed surface, normal to the fivefold axis. ${ }^{33-35}$ Crystallites with different orientations perpendicular to the growth direction grow more slowly and are kinetically hindered.
Nevertheless, production of atomically flat surfaces of bulk QC "single crystals" often requires annealing ${ }^{36}$ for some hours at temperatures around $700{ }^{\circ} \mathrm{C}$. Assuming terrace heights of the order of $0.5 \mathrm{~nm}$, the presence of Kiessig fringes in the reflectivity curves, even for film growth at temperatures as low as $300{ }^{\circ} \mathrm{C}$, indicates that our films cannot have fluctuations of more than \pm 10 terraces across the whole extent of the film. QCs differ from periodic structures in that the potential barrier across a step of a surface terrace will always raise the areal energy by an amount proportional to the length of the terrace perimeter, regardless of the temperature ${ }^{37}$ hence, step flow two-dimensional growth will be preferred over island nucleation and ripening, ${ }^{38}$ assuming there is sufficient surface diffusion, as provided by PLD, to allow migration to step edges.

\section{CONCLUSIONS}

In conclusion, the evolution of quasicrystalline order in highly textured $i$-TNZ films has been determined. An initial low-density amorphous buffer layer exhibiting only local icosahedral order has been identified that has a thickness of $\approx 5 \mathrm{~nm}$, corresponding to the size of the first inflation of the Bergman cluster. The transition from this to growth of denser material with long-range icosahedral order is characterized by a sudden drop in the deposition rate and by an increase in the x-ray critical angle. The final films consist of columnar grains with one of their fivefold axes pointing exactly perpendicular to the film surface. Their orientation is determined by minimization of the surface energy rather than any locking to the substrate net, which also accounts for the very low roughness, even for films with thicknesses in excess of $100 \mathrm{~nm}$.

\section{ACKNOWLEDGMENTS}

Support of this work by the Schweizerischer Nationalfonds zur Förderung der wissenschaftlichen Forschung is gratefully acknowledged. The authors would like to thank M. Lange and D. Meister for their assistance in construction of the deposition chamber, A. Al-Adwan and D. Maden for their help in software and interface development, and M. Döbeli for his assistance with the RBS measurements. P.R.W. thanks the Physical Chemistry Institute of the University of Zurich for financial support.
*Electronic address: philip.willmott@psi.ch

${ }^{1}$ D. Feuerbacher, P. Schall, Y. Estrin, and Y. Brechet, Philos. Mag. Lett. 81, 473 (2001).

${ }^{2}$ A. M. Viano, E. H. Majzoub, R. M. Stroud, M. J. Kramer, S. T. Misture, P. C. Gibbons, and K. F. Kelton, Philos. Mag. A 78, 131 (1998).

${ }^{3}$ Á. Csanády, K. Urban, J. Mayer, and P. B. Barna, J. Vac. Sci. Technol. A 5, 1733 (1987).

${ }^{4}$ P. B. Barna, G. Radnóczi, Á. Csanády, and K. Urban, Scr. Metall. 22, 373 (1988).

${ }^{5}$ P. B. Barna, Á. Csanády, G. Radnóczi, K. Urban, and U. Timmer,
Thin Solid Films 193-194, 1 (1990).

${ }^{6}$ L. Bendersky, Phys. Rev. Lett. 55, 1461 (1985).

${ }^{7}$ P. R. Willmott, R. Herger, and C. M. Schlepütz, Thin Solid Films 453-454, 436 (2004).

${ }^{8}$ P. R. Willmott, Prog. Surf. Sci. 76, 163 (2004).

${ }^{9}$ P. R. Willmott, in Applications of Pulsed Laser Deposition, 1st ed., edited by D. B. Chrisey and R. W. Eason (Wiley Interscience, New York, 2005).

${ }^{10}$ N. Ichikawa, O. Matsumoto, T. Hara, T. Kitahara, T. Yamauchi, T. Matsuda, T. Takeuchi, and U. Mizutani, Jpn. J. Appl. Phys., Part 2 33, L736 (1994). 
${ }^{11}$ V. Brien, A. Dauscher, P. Weisbecker, and F. Machizaud, Appl. Phys. A: Mater. Sci. Process. 76, 187 (2003).

${ }^{12}$ V. Brien, A. Dauscher, P. Weisbecker, J. Ghanbaja, and F. Machizaud, J. Cryst. Growth 256, 407 (2003).

${ }^{13}$ R. Timm, P. R. Willmott, and J. R. Huber, J. Appl. Phys. 80, 1794 (1996).

${ }^{14}$ P. R. Willmott, C. M. Schlepütz, B. D. Patterson, R. Herger, M. Lange, D. Meister, D. Maden, Ch. Brönnimann, E. F. Eikenberry, G. Hülsen, and A. Al-Adwan (unpublished).

${ }^{15}$ E. Vlieg, J. Appl. Crystallogr. 31, 198 (1998).

${ }^{16}$ B. D. Patterson, R. Abela, H. Auderset, Q. Chen, F. Fauth, F. Gozzo, G. Ingold, H. Kühne, M. Lange, D. Maden, D. Meister, P. Pattison, T. Schmidt, B. Schmitt, C. Schulze, M. Shi, M. Stampanoni, and P. R. Willmott (unpublished).

${ }^{17}$ R. M. Stroud, K. F. Kelton, and S. T. Misture, J. Mater. Res. 12, 434 (1997).

${ }^{18}$ J. B. Qiang, Y. Wang, D. H. Wang, M. Kramer, P. Thiel, and C. Dong, J. Non-Cryst. Solids 334-335, 223 (2004).

${ }^{19} \mathrm{http}: / /$ jcrystal.com/products/quaref/index.html

${ }^{20}$ K. F. Kelton, G. W. Lee, A. K. Gangopadhyay, R. W. Hyers, T. J. Rathz, J. R. Rogers, M. Robinson, and D. S. Robinson, Phys. Rev. Lett. 90, 195504 (2003).

${ }^{21}$ F. C. Frank, Proc. R. Soc. London 215, 43 (1952).

${ }^{22}$ W. J. Kim, P. C. Gibbons, K. F. Kelton, and W. B. Yelon, Phys. Rev. B 58, 2578 (1998).

${ }^{23}$ E. J. Widjaja and L. D. Marks, Thin Solid Films 420, 295 (2002).
${ }^{24}$ For our indexing system, we adopted the vector basis $\left\{q_{1} \cdots q_{6}\right\}$, with $q_{1}$ perpendicular to the substrate surface and $q_{2}$ to $q_{5}$ subtending an angle with $q_{1}$ equal to $63.43^{\circ}$, the dihedral angle of a dodecahedron.

${ }^{25}$ C. Janot and M. de Boissieu, Phys. Rev. Lett. 72, 1674 (1994).

${ }^{26}$ K. F. Kelton, Mater. Sci. Eng., A 375-377, 31 (2004).

${ }^{27}$ F. Seitz and J. S. Koehler, Solid State Phys. 2, 305 (1956).

${ }^{28}$ T. Diaz de la Rubia, R. S. Averbach, R. Benedek, and W. E. King, Phys. Rev. Lett. 59, 1930 (1987).

${ }^{29}$ B. Park, J. Appl. Phys. 82, 4219 (1997).

${ }^{30}$ R. G. Hennig, E. H. Mazjoub, A. E. Carlsson, K. F. Kelton, C. L. Henley, W. B. Yelon, and S. Misture, Mater. Sci. Eng., A 294296, 361 (2000).

${ }^{31}$ H.-C. Jeong and P. J. Steinhardt, Phys. Rev. Lett. 73, 1943 (1994).

${ }^{32}$ R. Mikulla, J. Stadler, F. Krul, H.-R. Trebin, and P. Gumbsch, Phys. Rev. Lett. 81, 3163 (1998).

${ }^{33}$ T. M. Schaub, D. E. Bürgler, H.-J. Güntherodt, and J. B. Suck, Phys. Rev. Lett. 73, 1255 (1994).

${ }^{34}$ K. Chattopadhyay, N. Ravishankar, and R. Goswami, Prog. Cryst. Growth Charact. Mater. 34, 237 (1997).

${ }^{35}$ L. Barbier, D. L. Floc'h, Y. Calvayrac, and D. Gratias, Phys. Rev. Lett. 88, 085506 (2002).

${ }^{36}$ J. Ledieu, V. R. Dhanak, R. D. Diehl, T. A. Lograsso, D. W. Delaney, and R. McGrath, Surf. Sci. 512, 77 (2002).

${ }^{37}$ C. L. Henley and R. Lipowsky, Phys. Rev. Lett. 59, 1679 (1987).

${ }^{38}$ J. Toner, Phys. Rev. Lett. 64, 930 (1990). 